\title{
Front Matter: Volume 11443
}

, "Front Matter: Volume 11443," Proc. SPIE 11443, Space Telescopes and Instrumentation 2020: Optical, Infrared, and Millimeter Wave, 1144301 (13 January 2021); doi: 10.1117/12.2591699

SPIE. Event: SPIE Astronomical Telescopes + Instrumentation, 2020, Online Only 


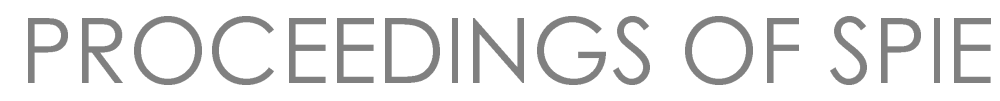

\title{
Space Telescopes and Instrumentation 2020: Optical, Infrared, and Millimeter Wave
}

\author{
Makenzie Lystrup \\ Marshall D. Perrin
}

Editors

14-22 December 2020

Online Only, United States

Sponsored and Published by

SPIE

Volume 11443

Part One of Two Parts 
The papers in this volume were part of the technical conference cited on the cover and title page. Papers were selected and subject to review by the editors and conference program committee. Some conference presentations may not be available for publication. Additional papers and presentation recordings may be available online in the SPIE Digital Library at SPIEDigitalLibrary.org.

The papers reflect the work and thoughts of the authors and are published herein as submitted. The publisher is not responsible for the validity of the information or for any outcomes resulting from reliance thereon.

Please use the following format to cite material from these proceedings:

Author(s), "Title of Paper," in Space Telescopes and Instrumentation 2020: Optical, Infrared, and Millimeter Wave, edited by Makenzie Lystrup, Marshall D. Perrin, Proceedings of SPIE Vol. 11443 (SPIE, Bellingham, WA, 2020) Seven-digit Article CID Number.

ISSN: 0277-786X

ISSN: 1996-756X (electronic)

ISBN: 9781510636736

ISBN: 9781510636743 (electronic)

Published by

SPIE

P.O. Box 10, Bellingham, Washington 98227-0010 USA

Telephone +1 3606763290 (Pacific Time) · Fax + 13606471445

SPIE.org

Copyright @ 2020, Society of Photo-Optical Instrumentation Engineers.

Copying of material in this book for internal or personal use, or for the internal or personal use of specific clients, beyond the fair use provisions granted by the U.S. Copyright Law is authorized by SPIE subject to payment of copying fees. The Transactional Reporting Service base fee for this volume is $\$ 21.00$ per article (or portion thereof), which should be paid directly to the Copyright Clearance Center (CCC), 222 Rosewood Drive, Danvers, MA 01923. Payment may also be made electronically through CCC Online at copyright.com. Other copying for republication, resale, advertising or promotion, or any form of systematic or multiple reproduction of any material in this book is prohibited except with permission in writing from the publisher. The CCC fee code is 0277$786 \times / 20 / \$ 21.00$.

Printed in the United States of America by Curran Associates, Inc., under license from SPIE.

Publication of record for individual papers is online in the SPIE Digital Library.

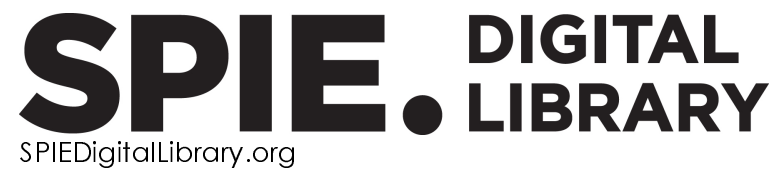

Paper Numbering: Proceedings of SPIE follow an e-First publication model. A unique citation identifier (CID) number is assigned to each article at the time of publication. Utilization of CIDs allows articles to be fully citable as soon as they are published online, and connects the same identifier to all online and print versions of the publication. SPIE uses a seven-digit CID article numbering system structured as follows:

- The first five digits correspond to the SPIE volume number.

- The last two digits indicate publication order within the volume using a Base 36 numbering system employing both numerals and letters. These two-number sets start with $00,01,02,03,04$, 05, 06, 07, 08, 09, OA, OB ... 0Z, followed by 10-1Z, 20-2Z, etc. The CID Number appears on each page of the manuscript. 


\title{
Contents
}

\section{Part One}

OPERATIONAL \& PAST MISSIONS

\begin{abstract}
1144308 The role of NASA Engineering and Safety Center (NESC) in advancing NASA's astrophysics missions (past, present, and future) [1 1 443-270]

1144309 The Herschel Space Observatory development, operation and post-operations: lessons learned [1 1 1443-1]

WIDE FIELD SURVEY MISSIONS I
\end{abstract}

11443 OF Euclid mission status after mission critical design [1 1443-7]

$114430 G \quad$ Status of the performance of the Euclid spacecraft [1 1 443-8]

WIDE FIELD SURVEY MISSIONS II

11443 Ol SPHEREx: NASA's near-infrared spectrophotometric all-sky survey [1 1443-10]

$114430 \mathrm{~J} \quad$ A space-based near-infrared sky survey to study the oxygen abundance in cool stars [1 1 443-11]

\section{ASTROMETRIC MISSIONS}

11443 ON Progress on the Astrometric Gravitation Probe design [1 $1443-17]$

$1144300 \quad$ The Micro-arcsecond Astrometry Small Satellite: MASS [1 1443-268]

11443 OP RAFTER: Ring Astrometric Field Telescope for Exo-planets and Relativity [11443-281]

\section{TELESCOPES FOR TRANSIENT FOLLOWUPS}

$114430 Q \quad$ The Visible Telescope onboard the Chinese-French SVOM satellite [1 1443-18] 
11443 OR Development of an optical and near-infrared telescope onboard the HiZ-GUNDAM mission [1 1 443-19]

JWST

11443 OT Status of the James Webb Space Telescope mission [1 1443-21]

11443 OW Preparing the JWST observatory for science observations [1 1443-24]

TRANSITING EXOPLANET MISSIONS I

1144310 The ESA Ariel mission is ready for implementation [1 1443-78]

$1144312 \quad$ PLATO telescope optical units: an update on working status [1 1443-80]

TRANSITING EXOPLANET MISSIONS II

1144314 CHEOPS, the ESA mission for exo-planets characterization: early operations and commissioning results [1 1 1443-82]

$1144316 \quad$ LOTUS: wide-field monitoring nanosatellite for finding long-period transiting planets [1 $1443-84]$

1144319 The MARSU CubeSat: monitoring the activity and planetary transits of low-mass stars and young solar analogues [1 $1443-87]$

ENABLING TECHNOLOGIES: OPTICS AND OPTOMECHANICS

11443 1B Optical space telescope without mirrors [1 1443-89]

11443 1D Programmable microshutter selection masks in application to UV spectroscopy [11443-91]

ENABLING TECHNOLOGIES: DETECTORS AND ELECTRONICS

$114431 \mathrm{~L} \quad$ MAJIS/JUICE VIS-NIR FM and SM detectors characterization [1 1443-137]

iv 
INTERFEROMETRY, FORMATION FLYING, AND STARSHADES

$114431 \mathrm{~N} \quad$ Far-infrared intensity interferometry for high angular resolution imaging [11443-139]

$114431 \mathrm{R} \quad$ Validation of diffraction models with experimental results from the Princeton starshade testbed [1 1443-143]

EXOPLANET IMAGING MISSIONS I

$114431 \mathrm{U} \quad$ The Nancy Grace Roman Space Telescope Coronagraph Instrument (CGI) technology demonstration [1 1 1443-194]

11443 IW Roman CGI testbed HOWFSC modeling and validation [1 1443-196]

$114431 Y \quad$ Design of the vacuum high contrast imaging testbed for CDEEP, the Coronagraphic Debris and Exoplanet Exploring Pioneer [1 $1443-198]$

EXOPLANET IMAGING MISSIONS II

1144312 A space mission dedicated for the characterization of habitable rocky planets [11443-199]

1144320 The Habitable Exoplanet Observatory mission concept [1 1443-200]

$1144324 \quad$ Integration time adjusted completeness [1 1443-204]

FAR-INFRARED AND MILLIMETER MISSIONS I

1144327 The 2020 SPICA telescope preliminary design and predicted performance [1 1443-207]

$1144328 \quad$ Cryogenic system of the infrared space mission SPICA [1 1443-208]

1144329 Mechanical cooler system for the infrared space mission SPICA [1 1443-209]

11443 2A ATSA: a cold, active telescope for Space Astronomy [1 1443-269]

FAR-INFRARED AND MILLIMETER MISSIONS III

$114432 \mathrm{~F} \quad$ LiteBIRD satellite: JAXA's new strategic L-class mission for all-sky surveys of cosmic microwave background polarization [1 1443-249] 
$114432 \mathrm{G}$ Overview of the medium and high frequency telescopes of the LiteBIRD space mission [1 1 443-250]

TECHNOLOGIES: WAVEFRONT SENSING

$114432 \mathrm{~K} \quad$ Implementation of a dark hole maintenance algorithm for speckle drift in a high contrast space coronagraph [1 1443-254]

$114432 \mathrm{~L} \quad$ Cross-fringe technique for sensing piston errors of segmented mirror telescope [1 1443-255]

TECHNOLOGIES: HIGH CONTRAST IMAGING

$114432 \mathrm{M} \quad$ Construction of EXIST (Exoplanet Imaging System Testbed) toward future space coronagraphs [1 1 443-256]

$1144320 \quad$ Experimental analysis of the achromatic performance of a vector vortex coronagraph [1 $1443-258]$

$114432 \mathrm{Q}$ Wavefront control experiments with a single mode fiber at the High-Contrast Spectroscopy Testbed for Segmented Telescopes (HCST) [1 1443-260]

SOLAR SYSTEM AND HELIOPHYSICS

$114432 \mathrm{U} \quad$ Scientific processing pipeline for ASPIICS coronagraph [1 1443-264]

$114432 \mathrm{~W} \quad$ Focus mechanism for EXOMARS mission: lessons learned from preliminary design to space flight model delivery [1 $1443-266]$

POSTER SESSION: CUBESATS AND SMALLSATS

$1144330 \quad$ First error budget of a deployable CubeSat telescope [1 $1443-95]$

$1144331 \quad$ High-resolution deployable CubeSat prototype [1 1443-96]

1144332 Non-contact vibration isolation technology demonstration on a CubeSat [1 1443-97]

POSTER SESSION: EXOPLANET IMAGING

1144337 The Roman exoplanet imaging data challenge: a major community engagement effort [1 1443-146]

vi 
1144338 A review of simulation and performance modeling tools for the Roman coronagraph instrument [1 1 443-1 48]

1144339 Prediction and evaluation of the image of the WFIRST coronagraph pupil at the shaped-pupil mask [1 1443-149]

\section{Part Two}

11443 3C Habitable-zone observatory (HabEx) baseline 4-m telescope design and predicted performance [1 1443-152]

POSTER SESSION: HIGH CONTRAST IMAGING

11443 3J Predicting contrast sensitivity to segmented aperture misalignment modes for the HiCAT testbed [11443-158]

$114433 \mathrm{~L} \quad$ A sequential optimization procedure designed for Lyot coronagraph aiming to realize high contrast direct imaging for exoplanets [1 1 443-161]

11443 3N Data processing for high-contrast imaging with the James Webb Space Telescope [1 1443-163]

11443 3P Exploiting symmetries and progressive refinement for apodized pupil Lyot coronagraph design [1 $1443-165]$

11443 3R Design of the life signature detection polarimeter LSDpol [1 1443-167]

11443 3T ExoSpec project: an exoplanet spectroscopy technology research collaboration based at NASA's Goddard Space Flight Center and Ames Research Center [1 $1443-169]$

$114433 \mathrm{Y}$ Towards high throughput and low-order aberration robustness for vortex coronagraphs with central obstructions [1 1443-174]

$1144341 \quad$ Mueller matrix maps of dichroic filters reveal polarization aberrations [1 1443-275]

POSTER SESSION: WAVEFRONT SENSING AND CONTROL

$1144342 \quad$ Phasing a sparse telescope [1 1443-182]

$1144346 \quad$ Estimating low-order aberrations through a Lyot coronagraph with a Zernike wavefront sensor for exoplanet imaging [1 1443-186]

1144348 Integrating bias and gain invariance with the generalized Anscombe transform for wavefront sensing [1 1 1443-188]

1144349 Deep neural networks to improve the dynamic range of Zernike phase-contrast wavefront sensing in high-contrast imaging systems [1 1443-189] 
POSTER SESSION: JWST

$114434 \mathrm{D} \quad$ Modelling the path length of aluminium seen by the detectors in the MIRI instrument on the JWST [1 1 443-32]

\section{POSTER SESSION: TRANSITING EXOPLANET MISSIONS}

$114434 \mathrm{P}$ The role of the instrument control unit within the ARIEL Payload and its current design [1 1443-48]

$114434 \mathrm{Q}$ On the optical alignment of the PLATO cameras [1 1443-49]

$114434 \mathrm{R} \quad$ Product assurance for the PLATO Telescope optical unit [1 1443-50]

$114434 S$ A combined software and hardware data compression approach in PLATO [1 1443-51]

$114434 \mathrm{~T} \quad$ A white light collimator for Plato camera integration support [1 1443-52]

$114434 \mathrm{~V}$ The instrument control unit of the PLATO payload: design consolidation following the preliminary design review by ESA [1 1443-54]

$114434 \mathrm{X}$ Design and validation of the boot software for the instrument control unit of the PLATO mission [1 1 443-56]

$1144351 \quad$ Design of the electrical ground support equipment for the PLATO camera AIV [1 1443-60]

POSTER SESSION: WIDE FIELD SURVEY MISSIONS

1144359 Data processing unit's hardware and application software description of the Near Infrared Spectro-Photometer: Euclid mission [1 1443-69]

$114435 \mathrm{~A} \quad$ Pre-flight optical test and calibration for the Cosmic Infrared Background ExpeRiment 2 (CIBER-2) [1 1443-70]

$114435 \mathrm{~B} \quad$ The application software for the instrument control unit of the NISP instrument of the Euclid mission: final status and lessons learned after delivery of the flight version [1 $1443-71$ ]

$114435 \mathrm{~F} \quad$ Photon counting and precision photometry for the Roman Space Telescope Coronagraph [1 1 443-271] 
POSTER SESSION: DETECTORS AND ELECTRONICS

$114435 \mathrm{H} \quad$ Multicore processor based instrument control and data processing units design for the SPICA instruments [1 1 1443-104]

$114435 \mathrm{M} \quad$ MVIC flight and flight spare sensor calibration [1 1443-109]

$114435 \mathrm{D}$ Detector fabrication development for the LiteBIRD satellite mission [1 $1443-114]$

POSTER SESSION: OPTICS AND OPTOMECHANICS

1144365 Parameters for mirror selection: trades between glass ceramics, glass, metals, ceramics and cordierites [1 $1443-130]$

POSTER SESSION: INTERFEROMETRY, FORMATION FLYING, AND STARSHADES

$1144366 \quad$ Linear formation-flying astronomical interferometry in low-Earth orbit: a feasibility study [1 1 443-177]

$114436 \mathrm{~A} \quad$ Broadband characterization of anti-reflection coated starshade optical edges for solar glint control [11443-181]

\section{POSTER SESSION: FAR-IR AND MILLIMETER}

11443 6B Development of EXo-Zodiacal Infrared Telescope (EXZIT) for observation of visible and nearinfrared extragalactic background light [1 1443-2 10]

11443 6C Polarization angle measurement of LiteBIRD low frequency telescope scaled model [1 $1443-211]$

$114436 \mathrm{D} \quad$ Laboratory experiment of densified pupil spectrograph for the Origins Space Telescope [1 1443-212]

$114436 \mathrm{~F} \quad$ Simulating electromagnetic transfer function from the transmission antennae to the sensors vicinity in LiteBIRD [1 $1443-214]$

114436 SPICA Mid-infrared Instrument (SMI): The latest design and specifications [1 1443-215]

$114436 \mathrm{H} \quad$ The calibration source assembly for SPICA/SAFARI instrument [1 1443-216]

$1144361 \quad$ The 4K focal plane unit for SPICA's SAFARI far infrared instrument [1 1443-217]

$114436 \mathrm{~K}$ The mid-infrared spectrometer/camera (MISC) for the Origins Space Telescope [1 1443-219] 
$114436 \mathrm{~L} \quad$ Calibration strategy for the SPICA/SAFARI instrument [1 1 443-220]

$114436 R \quad$ A far infrared spectrometer for SPICA mission: optical E2E of SAFARI [1 1443-226]

11443 6X Evaluation of reconstructed angular error of a continuous rotating HWP for LiteBIRD [1 1443-276]

$114436 Z$ A polarization modulator unit for the mid- and high-frequency telescopes of the LiteBIRD mission [1 1443-282]

$1144370 \quad$ The optical design of the Litebird middle and high frequency telescope [1 1443-283]

POSTER SESSION: SOLAR SYSTEM AND HELIOPHYSICS

1144373 Selection of in-flight flat-field calibration dither pattern for the Solar Disk Imager of the Lymanalpha Solar Telescope [1 1443-234]

1144374 Setting the parameters for the stellar calibration of the SIMBIO-SYS STC camera on-board the ESA BepiColombo mission [1 1 443-235]

1144378 On-board de-spiking implemented by MAJIS, the VIS/NIR imaging spectrometer of JUICE [1 1 443-239]

1144379 The optical head of the EnVisS camera for the Comet Interceptor ESA mission: phase 0 study [1 $1443-240]$

11443 7C HYPSOS: a HYPerspectral stereo observing system for solar system exploration [1 1443-243]

11443 7E Simulations of stray light from the surface scattering of the Solar Corona Imager primary mirror [1 1 443-272]

$114437 G \quad$ Thermal-vacuum and security system of the characterization facility for MAJIS/JUICE VIS-NIR FM and SM detectors [1 1443-277]

11443 7H Characterization facility for the MAJIS/JUICE VIS-NIR FM and SM detectors [1 1443-278]

POSTER SESSION: MISSION DEVELOPMENT AND PLANNING

$1144371 \quad$ Establishing a resilient flagships science program [1 1443-44] 